\title{
NUEVOS TAXA DE HELECHOS ARBORESCENTES (FILICALES: CYATHEACEAE) EN COSTA RICA
}

\author{
Alexánder Fco. Rojas Alvarado \\ Jardín Botánico Lankester, Universidad de Costa Rica, apdo. 1031-7050, Cartago, Costa Rica. \\ afrojasa@yahoo.com
}

\begin{abstract}
New species are described and new combinations are made in the fern genera Cnemidaria and Cyathea from Costa Rica. Cnemidaria chiricana (Maxon) A. Rojas is considered here a species different from C. mutica (H. Christ) R.M. Tryon, and C. mutica var. contigua (Maxon) Stolze is included as a variety of the former. Cyathea povedae A. Rojas is here described as a new species and compared to C. fulva (M. Martens \& Galeotti) Fée. The hybrid between Cyathea stolzei A.R. Sm. ex Lellinger and C. ursina (Maxon) Lellinger mentioned by Smith \& Grayum (1988) is here described.
\end{abstract}

Resumen. Cnemidaria chiricana (Maxon) A. Rojas se considera una especie diferente a C. mutica (H. Christ) R.M. Tryon; $C$. mutica var. contigua (Maxon) Stolze se trata como una variedad de $C$. chiricana. Cyathea povedae A. Rojas se describe como especie nueva y se compara con C. fulva (M. Martens \& Galeotti) Fée. Además, aquí se describe el híbrido entre Cyathea stolzei A.R. Sm. ex Lellinger y C. ursina (Maxon) Lellinger, mencionado por Smith \& Grayum (1988).

Palabras clave / Kew words: Cnemidaria, Cyathea, Cyatheaceae, tree ferns / helechos arborescentes, Pteridophyta, Costa Rica.

Tryon (1970) divide la familia Cyatheaceae con base en caracteres de las escamas, presencia o ausencia de indusio y venación. Así, caracteriza a Cnemidaria C. Presl por tener escamas no conformes y no setadas y lámina con venas reticuladas, y a Cyathea $\mathrm{Sm}$. por tener escamas no conformes y no setadas, lámina sin venas reticuladas y soros indusiados. Estos soros no indusiados son característicos de Trichopteris Spreng., un sinónimo de Cyathea, como fue indicado por Lellinger (1987).

Stolze (1974) define al género Cnemidaria como plantas con rizoma compacto (no arborescente), frondas pinnadas a pinnado-pinnatífidas, venas costales comúnmente formando areolas y esporas con poros ecuatoriales. Este concepto es apoyado por otros autores, como Lellinger (1989) y Moran (1995), en tanto que Cyathea es caracterizada por incluir plantas con tallo comúnmente arborescente, frondas principalmente bipinnado-pinnatífidas (raramente menos divididas), venas costales o costulares no formando areolas y esporas sin poros ecuatoriales (Tryon 1976, Lellinger 1989, Moran 1995).

Cnemidaria. Stolze (1974) considera que todas las especies de nervadura libre presentes en Costa Rica son, en realidad, variedades de Cnemidaria mutica $(\mathrm{H}$. Christ) R.M. Tryon, e indica que las variaciones en tamaño de las frondas, incisión de las pinnas y ápice de los segmentos son caracteres traslapados. Como puede observarse en la clave del género Cnemidaria de Costa Rica, las variedades mencionadas se agrupan de mejor forma en dos especies, cada una con dos variedades; con ello se reconoce nuevamente el nombre $C$. chiricana (Maxon) R.M. Tryon (sensu Tryon 1970). La combinación C. mutica var. contigua (Maxon) Stolze (Stolze 1974) es, así, sinónimo de C. chiricana var. contigua.

Cnemidaria chiricana (Maxon) R.M. Tryon var. contigua (Underw. ex Maxon) A. Rojas, comb. nova

Hemitelia contigua Underw. ex Maxon, Contr. U.S. Natl. Herb. 16: 32. 1912. Tipo: Costa Rica, 5 mi. S of Cartago, Maxon 523 (NY!, frag. US).

Cyathea contigua (Underw. ex Maxon) Domin, Pteridophyta: 263. 1929.

Cnemidaria contigua (Underw. ex Maxon) R.M. Tryon, Contr. Gray Herb. 200: 52. 1970.

Cnemidaria mutica (H. Christ) R.M. Tryon var. contigua (Maxon) Stolze, Fieldiana, Bot. 37: 37-38. 1974.

Tallo compacto, de $2-5 \mathrm{~cm}$ de diámetro; frondas de 1.2-1.6 x 0.3-0.5 m; estípite estramíneo, liso o con espinas diminutas, la base escamosa, las escamas 515 × 2-4 mm, lanceoladas, bicoloras, con un ancho margen blanco, o casi concoloras y blanquecinas, con una franja pardo-clara a negruzca; lámina pinnado- 
pinnatífida, reducida gradualmente a un ápice pinnatífido; raquis glabro, o muy dispersamente peloso abaxialmente; pinnas basales $5-8 \times 1.5-2.0 \mathrm{~cm}$, pinnas medias $18-25$ x 2.5-4.0 (-5.0) cm, incisas $1 / 2-3 / 4$ hacia la costa, sésiles a cortamente pediculadas; segmentos subenteros (excepto en los ápices serrulados), obtusos a raramente subagudos, unidos por senos agudos, angostos; segmentos no más de 1.5 veces más largos que anchos más allá del seno; nervaduras 7-10 (-12) pares por segmento, una a dos veces bifurcadas; tejido laminar entre las nervaduras glabro; soros inframedios a supramedios, en una sola línea entre el margen del segmento y la cóstula; indusios casi semicirculares, en el lado costular del receptáculo.

HáBitat. Bosque muy húmedo premontano y bosque pluvial premontano.

Distribución. Cordillera Central y región norte de la Cordillera de Talamanca, Costa Rica, 1200-2200 m. Variedad endémica.

EJemplar Representativo. A. Rojas 1076 (CR, INB, $\mathrm{MO})$.

\section{Clave de las especies de Cnemidaria de Costa Rica}

1. Nervaduras de la base entre los segmentos libres .2

1. Nervaduras de la base entre los segmentos comúnmente fusionándose en areolas costales .5

2. Frondas de 1.2-1.6 (-2) x 0.3-0.6 m; estípite de 1/5-1/12 de la longitud de la fronda; pinnas medias de 18-30 x 2.5-4 (-5) cm; segmentos de las pinnas medias 1-2 veces más largos que anchos; pinnas incisas de 1/2-3/4 hacia la costa; venas simples a bifurcadas .....

2. Frondas de 2-3 m x (0.5-) 0.8-1.4 m; estípite de 1/3-1/5 de la longitud de la fronda; pinnas medias de 30-70 x (-5) 7-25 cm, segmentos de las pinnas medias (2-) 3-8 veces más largos que anchos; pinnas incisas más de 3/4 hacia la costa; venas $2-5$ veces bifurcadas o pinnadas.

3. Estípite no espinoso; raquis peloso en ambas superficies, segmentos con el ápice obtuso a agudo. 3. Estípite comúnmente espinoso; raquis glabro o dispersamente peloso sólo en el lado abaxial, segmentos con el ápice agudo a acuminado.. C. chiricana var. contigua

4. Segmentos basales de las pinnas medias libres o al menos separados y unidos por una costa alada; segmentos de las pinnas medias lobados a crenados, con la nervadura de los lóbulos comúnmente pinnada.

..C. mutica var. grandis

4. Segmentos basales de las pinnas sésiles; segmentos de las pinnas medias enteros, con la nervadura comúnmente 2 a 4 veces bifurcada. C. mutica var. mutica

5. Pinnas incisas aprox. 7/8 o raramente las pinnas basales libres; segmentos con el ápice agudo a acuminado; areolas costulares a menudo presentes C. horrida

5. Pinnas incisas 1/2-7/8; segmentos con el ápice obtuso o redondeado; areolas costulares ausentes. .6

6. Pinnas medias de $18-30 \mathrm{~cm}$ de largo, incisas $1 / 3-1 / 2$ hacia la costa C. choricarpa

6. Pinnas medias de $33-47 \mathrm{~cm}$ de largo, incisas 1/2-7/8 hacia la costa. ..C. cocleana

Cyathea. Rojas (2001) indica que Cyathea fulva (M. Martens \& Galeotti) Fée es un grupo complejo, del cual separa C. onusta H. Christ e indica que el material de Costa Rica, Panamá, Colombia y Venezuela puede corresponder a otra especie más relacionada con $C$. caracasana (Klotzsch) Domin.

\section{Cyathea povedae A. Rojas, sp. nova}

TIPo: COSTA RICA. San José: Dota, Zona Protectora
Cerro Nara, 9²9’40" N, 8400'50” W, 900-1000 m, 23 jul 1998, A. Estrada et al. 1674 (Holotipo: CR). Fig. 1.

A Cyathea fulva (M. Martens \& Galeotti) Fée similis, sed stipitis squamis pardalibus (vs. aureis), laminae costulae venaeque squamis albidis (vs. luteopardalibus vel pardalibus), planis (vs. bulliformibus) differt. 
Tallo de 2-7 m de alto, de 8-12 cm de diámetro, con frondas caedizas dejando cicatrices; frondas de (2-) 2.5 $3.5 \mathrm{~m}$ de largo; estípite espinoso, pajizo a pardo, opaco, con caspilla parda; escamas del estípite de (10-) 15-30 x 2-5 mm, lanceoladas, concoloras o casi concoloras, pardas a pardo-oscuras, a veces con un angosto margen más claro; lámina bipinnado-pinnatífida a tripinnada, reducida gradualmente a un ápice pinnatífido; raquis glabro a escuamuloso, con caspilla parda a amarillenta formada de escuámulas diminutas; pinnas sésiles o pediculadas, el pedículo de $0-1(-1.5) \mathrm{cm}$; pinnas medias (-40) 50-75 (-85) x (12-) $17-24 \mathrm{~cm}$, lanceoladooblongas; costas glabras o casi glabras, a menudo dispersamente cubiertas de diminutas escuámulas amarillentas a pardo-amarillentas mezcladas con tricomas hialinos de 0.1-0.3 mm; pínnulas (6-) 8-12 x (1.2-) $1.5-2.6 \mathrm{~cm}$, sésiles a cortamente pediculadas, el pedículo hasta $2 \mathrm{~mm}$; cóstulas glabras a puberulentas y escamosas, los tricomas de 0.1-0.3 mm, hialinos a amarillentos, generalmente adpresos, las escamas anchamente ovadas, pálidas a amarillentas; segmentos de las pínnulas medias 12-20 pares, 3-5 mm de ancho, enteros a serrulados; vena media de las pínnulas glabra o con pelos hialinos patentes sólo en la parte terminal del haz, glabra a medianamente pilosa y escamosa, las escamas 1-3 mm de largo, ovadas, con ápice agudo a caudado, blanquecinas a amarillentas, planas (excepto en el ápice de los segmentos con escamas a veces levemente abolladas), con pelos de 0.5-2 mm de largo, patentes, hialinos, a veces también con pelos de 0.2-0.5 $\mathrm{mm}$ de largo, hialinos a pardos, adpresos, retorcidos; nervaduras 8-10 pares por segmento, bifurcadas, glabras o dispersamente pilosas en ambas superficies, los pelos 0.2-0.5 mm, blanquecinos a pardo-amarillentos, patentes, abaxialmente a menudo mezclados con pelos 0.1-0.3 mm, adpresos, retorcidos; tejido laminar entre las nervaduras glabro o con pelos de 0.2-0.5 mm, blanquecinos, patentes; soros subcostales; paráfisos más cortos que los esporangios, inconspicuos; indusio globoso, que rodea completamente al receptáculo, evanescente en la madurez.

HÁBitat. Bosque muy húmedo tropical, transición a premontano, bosque pluvial premontano y bosque pluvial montano.

DistriBución. Cordillera de Tilarán, vertiente norte de la Cordillera Central, Cordillera de Talamanca y serranías del Pacífico, 500-1600 m. Especie conocida sólo en Costa Rica.

Paratipos: COSTA RICA. Alajuela: Alajuela, Cordillera Central, Cariblanco, camino a Virgen del
Socorro, $10^{\circ} 15^{\prime} 25^{\prime \prime} \mathrm{N}, 8^{\circ} 10^{\prime} 20^{\prime \prime} \mathrm{W}, 720-870 \mathrm{~m}, 11$ sept 1994, A. Rojas 1429 (CR, INB, MO). Cartago: near Moravia and along the road toward Turrialba, $9^{\circ} 51^{\prime} \mathrm{N}, 83^{\circ} 26^{\prime} \mathrm{W}, 1000 \mathrm{~m}, 19-20$ Dec 1966, W. Burger \& W. Ramírez 3964 (CR, NY). Limón: Limón, Valle de la Estrella, Reserva Indígena Chirripó, Chirripó Abajo, Almirante, $9^{\circ} 46^{\prime} \mathrm{N}, 83^{\circ} 20^{\prime} \mathrm{W}, 1060-1330 \mathrm{~m}$, 10 ago 1995, A. Rojas 2212 (INB, MO). Puntarenas: Coto Brus, Zona Protectora Las Tablas, Cordillera de Talamanca, Las Alturas de Cotón, Estación Biológica Las Alturas, sendero a Cerro Echandi, postes 1-7, 8 $57^{\prime} 15^{\prime \prime} \mathrm{N}, 82^{\circ} 50$ '10”'W, 1580-1620 m, 19 dic 1993, $A$. Rojas 719 (CR, INB). San José: Dota, Zona Protectora Cerro Nara, 9²9'40”N, 8400'50”'W, 800-900 m, 3 dic 1997, A. Estrada et al. 1387 (CR).

Otros especímenes estudiados: COSTA RICA. Alajuela: San Ramón, Los Ángeles, Reserva Biológica Monteverde, Pocosol, $10^{\circ} 17^{\prime} \mathrm{N}, 84^{\circ} 10^{\prime} \mathrm{W}, 720 \mathrm{~m}$, 19 Feb 1992, E. Bello 4401 (INB); Reserva Forestal San Ramón, N of the station, $c a .2 \mathrm{~km}, 10^{\circ} 12^{\prime} 40^{\prime \prime} \mathrm{N}$, 86³6'20"W, 1000-1300 m, 28 Mar 1991, J. Bittner 891 (CR), 13 Feb 1992, J. Bittner 1260 (CR); Reserva

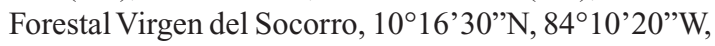
720 m, 10 Jan 1993, J. Bittner 1664 (CR, INB), J. Bittner 1665 (CR); Reserva Forestal San Ramón, trail on $\mathrm{S}$ of the field station, $10^{\circ} 12^{\prime} 40^{\prime \prime} \mathrm{N}, 86^{\circ} 36^{\prime} 20^{\prime \prime} \mathrm{W}$, 1050 m, 6 Feb 1993, J. Bittner 1683 (CR), Cariblanco, 800 m, 3 dic 1964, C. Nisman 113 (CR); 4 km SE de Fortuna, $2.5 \mathrm{~km} \mathrm{SW}$ sobre brecha, $10^{\circ} 30^{\prime} \mathrm{N}, 85^{\circ} 00^{\prime} \mathrm{W}$, ca. 500 m, 29 abr 1983, B. Pérez et al. 371 (CR); Upala, Bijagua, Finca Zapote, $10^{\circ} 45^{\prime} \mathrm{N}, 85^{\circ} 04^{\prime} \mathrm{W}$, 500-530 m, 9 jul 1994, A. Rojas 1279 (INB). Cartago: Turrialba, 800 m, 17 Jun 1932, W. Kupper 1593 (CR); Tapantí, 22 sept 1964, C. Nisman 17 (CR). Limón: Limón, Cerro Muchilla, Fila Matama, Cordillera

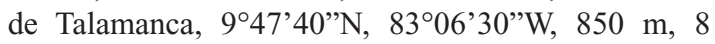
abr 1989, R. Robles \& A. Chacón 2726 (CR, INB). Puntarenas: About $2 \mathrm{~km} \mathrm{SE}$ of Monteverde, $10^{\circ} 18^{\prime} \mathrm{N}$,

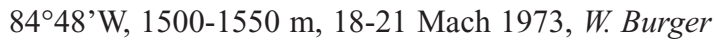
\& J. Gentry 8677 (CR, F); upper Río Burú, $2010 \mathrm{~m}$, 19 Aug 1983, L. Gómez et al. 21779 (CR); drenaje del Río Coto Brus, 1470 m, 22 feb 1965, C. Nisman 150 (CR); Coto Brus, San Vito de Java, Jardín Botánico Las Cruces, 1400 m, 14 dic 1982, B. Pérez \& C. Wille 241 (CR), 15 dic 1982, B. Pérez \& C. Wille 253 (CR); Coto Brus, Zona Protectora Las Tablas, Cordillera de Talamanca, Las Alturas de Cotón, Estación Biológica Las Alturas, sedero a Cerro Echandi, postes 1-7, $8^{\circ} 57^{\prime} 15^{\prime \prime} \mathrm{N}, 82^{\circ} 50$ '10’'W, 1580-1620 m, 19 dic 1993, A. Rojas 767 (INB). San José: ca. 1000 m, along Rt. 2, $11.6 \mathrm{~km}$ N of San Isidro del General, 16 Jun 1989, D. 
Conant 4412 (CR), Tarrazú, San Lorenzo, Naranjillo, camino a Quepos, 9 ${ }^{\circ} 33^{\prime} \mathrm{N}, 84^{\circ} 02^{\prime} \mathrm{W}, 600-700 \mathrm{~m}, 31$ jul 1998, A. Rojas et al. 4711 (INB).

Cyathea povedae difiere de Cyathea fulva (M. Martens \& Galeotti) Fée por tener un tallo más delgado $(8-12 \mathrm{~cm}$ de diámetro vs. $12-16 \mathrm{~cm})$, frondas caedizas dejando cicatrices al morir (vs. persistentes dejando la base de los estípites); escamas del estípite pardas a pardo oscuro (vs. amarillentas a pardo-rojizas) y escamas del tejido, costas y vena central de las pinnas secundarias blanquecinas (vs. pardo-amarillentas a pardas), planas (vs. abolladas) con las paredes laterales (las que se unen a las células adyacentes) muy resaltadas (vs. poco evidentes), pelos hialinos en las cóstulas pero no en las venas (vs. en las cóstulas y las venas) y tejido laminar glabro o piloso abaxialmente (vs. siempre glabro, Figs. 1-3). Por las escamas blancas y aplanadas en la lámina se parece a Cyathea pallescens (Sodiro) Domin, de Colombia, Ecuador y Perú, pero difiere por escamas del estípite planas (vs. crespas), pardo-oscuras y concoloras (vs. blanquecinas a pardas, cuando son pardas entonces bicoloras), escamas de las costas secundarias y terciarias sin escamas estrelladas (vs. con escamas estrelladas), tejido laminar a menudo con tricomas blanquecinos patentes (vs. sin ellos) y distribución altitudinal diferente [500-1600 m vs. (1500-) 2200-3300 m].

Por las escamas del estípite doradas y las del tejido laminar abolladas, Cyathea fulva parece estar muy relacionada con C. suprastrigosa (H. Christ) Maxon, de la cual difiere principalmente por la ausencia de pinnas subafleboides (vs. comúnmente presentes), escamas de la lámina completamente pardas, con ápice acuminado y entero (vs. ápice blanquecino y ancho, a menudo ramificado en cilios) y ausencia de pelos en el haz de la lámina (vs. pelos estrigosos en el haz). C. povedae parece estar más emparentada con $C$. onusta $\mathrm{H}$. Christ por las escamas del estípite pardas a pardo-oscuras y las escamas de la lámina blanquecinas; difiere de $C$. onusta por tener un tallo de 8-12 cm de diámetro [vs.
5-8 (-10) cm], frondas de (2-) 2.5-3.5 m de largo (vs. 1-2 m), estípite escamoso sólo en la base (vs. escamoso a todo su largo), raquis no escamoso (vs. escamoso en el dorso), pinnas secundarias de (6-) 8-13 cm de largo (vs. 4-7 cm de largo), segmentos de (8-) 10-15 x 3$5 \mathrm{~mm}$ (vs. segmentos de 5-8 $\times$ 2-3 mm), nervaduras glabras a dispersamente escamosas (vs. densamente escamosas) y las escamas planas (vs. abolladas).

Cyathea fulva habita en México y Centroamérica hasta Nicaragua. Aparentemente, los registros sudamericanos de esta especie corresponden a determinaciones erróneas de Cyathea aurea Klotzsch, C. caracasana (Klotzsch) Domin (s. l.) o C. delgadii Sternb., principalmente, junto con algunas entidades no reconocidas taxonómicamente de C. caracasana, que requieren de más estudios. Incluso las variedades de Cyathea caracasana probablemente corresponden a especies válidas, pero los especímenes fragmentarios y la escasez de información sobre características del tallo, así como sobre el tamaño de las frondas, hacen difícil la delimitación de estos taxa. Al menos Cyathea caracasana var. maxonii (Underw.) R.M. Tryon es notablemente diferente y geográficamente está separada.

Una característica que ayuda a diferenciar Cyathea fulva, C. povedae y C. caracasana (de Sudamérica) son los pelos blanquecinos, patentes y largos; en las pínnulas de C. fulva los pelos están restringidos sólo a las cóstulas, en $C$. povedae los pelos pueden estar en cóstulas, venas y tejido laminar, y en C. caracasana se hallan en cóstulas y venas únicamente, o no existen. Un caso crítico en la delimitación de Cyathea caracasana es C. caracasana var. boliviensis (Rosenst.) R.M. Tryon, ya que presenta pelos largos como los de $C$. delgadii, pero en menor densidad y con escamas abundantes sobre las cóstulas.

Etimología. Esta especie es dedicada al reconocido dendrólogo costarricense Luis Poveda, por sus innumerables contribuciones a la flora de Costa Rica.

\section{Clave de las especies de Cyathea con indusio globoso en Costa Rica}

1. Caspilla del estípite blanca. ..C. divergens var. divergens

1. Caspilla del estípite parda o ausente...... .2

2. Estípite atropurpúreo basalmente, castaño distalmente, brillante, inerme; caspilla ausente; plantas generalmente epífitas; pínnulas pediculadas, el pedículo 4-8 $\mathrm{mm}$. C. gracilis

2. Estípite pardo-amarillento a pardo oscuro, opaco, espinoso; caspilla presente; plantas terrestres o raramente $(C$. divergens) epífitas; pínnulas sésiles o pediculadas, el estípite hasta $3 \mathrm{~mm}$. .3 
3. Escamas del pecíolo doradas a pardo-amarillentas, haz y envés con muchos pelos blanquecinos patentes......4

3. Escamas del pecíolo pardas a pardo oscuro; haz y envés con pelos blanquecinos dispersos o ausentes...........5

4. Estípite comúnmente con pinnas subafleboides en la base; escamas de la cóstula y la vena central de los segmentos blanquecinas a amarillentas (raro ambarinas), abundantes; tejido laminar entre las nervaduras piloso; (2200-) 2500-3000 m. C. suprastrigosa

4. Estípite sin pinnas subafleboides en la base; escamas de la cóstula y la vena central de los segmentos pardas, escasas a ausentes; tejido laminar entre las nervaduras glabro; 300-1200 (-1600) $\mathrm{m}$. C. delgadii

5. Escamas costulares color ámbar a pardas. C. caracasana var. maxonii

5. Escamas costulares blancas a amarillentas. . .6

6. Frondas de 1-2 m, estípite escamoso a todo lo largo; raquis escamoso en el dorso; pínnulas 4-7 cm de largo; segmentos de 5-8 x 2-3 mm; nervaduras densamente escamosas. C. onusta

6. Frondas de (2-) 2.5-3.5 m, estípite escamoso sólo en la base; raquis no escamoso; pínnulas (6-) 8-13 cm de largo; segmentos de (8-) 10-15 x 3-5 mm; nervaduras glabras a dispersamente escamosas. C. povedae

Rojas (2001) menciona erróneamente la existencia en Costa Rica de Cyathea caracasana var. boliviensis y de C. c. var. meridensis (H. Karst.) R.M. Tryon, al interpretar inapropiadamente la clave de Tryon (1976), debido a que en Costa Rica existen tres formas reconocibles de $C$. caracasana var. maxonii. Una de ellas se halla en la Cordillera de Tilarán y en la Cordillera Central; se caracteriza por las escamas del estípite ovado-lanceoladas, pardo-oscuras y casi concoloras, con un leve margen más claro, segmentos cortos y anchos, tejido laminar coriáceo y pardo oscuro al secar. La forma típica tiene escamas del estípite pardo-amarillentas, con la base y el margen pardo oscuro, segmentos largos y angostos, tejido laminar cartáceo y pajizo a pardo amarillento al secar. Finalmente, existe una variedad en zonas altas de la Cordillera de Talamanca, que difiere de la variedad típica por las escamas del estípite pardas a pardo-rojizas y pelos estrigosos en el tejido laminar; se asemeja a $C$. suprastrigosa, pero con escamas de las cóstulas pardooscuras y escasas.

En la zona norte de Costa Rica, Smith \& Grayum (1988) descubrieron plantas que presentan características intermedias entre C. stolzei A.R. Sm. ex Lellinger y C. ursina (Maxon) Lellinger, al comparar en un cuadro y en ilustraciones varios caracteres, como escamas del estípite y del raquis, número de pinnas, incisión de las pinnas, entre otros caracteres, que evidencian un proceso de hibridación.

\section{Cyathea $\mathbf{x}$ smithiana A. Rojas, nothosp. nova}

TIPO: COSTA RICA. Heredia: Sarapiquí, La Guaria, Cerros Los Arrepentidos, entrando por el cementerio,
Tecnoforest del Norte S. A., $10^{\circ} 28^{\prime} 10^{\prime \prime} \mathrm{N}, 8^{\circ} 01^{\prime} 40^{\prime \prime} \mathrm{W}$, 100-160 m, 6 jul 1998, A. Rojas \& A. Soto 4693 (Holotipo: CR, isotipos: INB, MO, NY). Figs. 2 y 3.

Tallo hasta $0.3 \mathrm{~m}$ de alto, $1-3 \mathrm{~cm}$ de diámetro; frondas de 1-1.3 m de largo; estípite dispersamente muricado, pardo-amarillento a pardo, puberulento, los pelos menos de $0.2 \mathrm{~mm}$, blanquecinos a pardoamarillentos; escamas del estípite 5-10 x 1.0-2.5 mm, linear-lanceoladas, bicoloras, con un delgado margen blanco; caspilla ausente; lámina pinnado-pinnatífida, reducida gradualmente a un ápice pinnatífido; raquis similar al estípite en indumento; pinnas incisas $c a$. $1 / 2$ entre el margen y la costa, sésiles o escasamente pediculadas; pinnas medias 10-14 x 2.5-3.0 cm; costas glabrescentes o puberulentas, dispersamente escamosas, las escamas 1.5-3.0 x 0.3-1.0 mm, ovadas a lanceoladas, pardas; segmentos de las pinnas medias 15-20 pares, 4-5 $\mathrm{mm}$ de ancho, enteros; nervaduras 58 pares por segmento, simples o una vez bifurcadas; tejido laminar entre las nervaduras glabro; soros medios o ligeramente supramedios; paráfisos más largos que los esporangios, conspicuos, blanquecinos o hialinos; indusio escuamiforme, adpreso a la superficie de la lámina.

Hábitat. Bosque muy húmedo tropical y bosque muy húmedo tropical, transición a premontano. DistribuCión. Vertiente norte de la Cordillera Central, Costa Rica, 100-1000 m. Híbrido endémico.

PARATIPos: COSTA RICA. Heredia: Cerros Sardinal, ca. $2 \mathrm{~km} \mathrm{~N}$ of Chilamate de Sarapiquí (Finca La Martita), $10^{\circ} 28^{\prime} \mathrm{N}, 84^{\circ} 04^{\prime} \mathrm{W}$, ca. 80-160 m, A. Smith 
et al. 1780 (CR, MO, UC).

Etimología. Este híbrido es dedicado a Alan Reid Smith, quien lo notó y lo comunicó primeramente, junto con Michael H. Grayum, pero sin asignarle un nombre científico.

Agradecimientos. A Carlos O. Morales por la corrección del latín, a los curadores del Herbario Nacional de Costa Rica (CR), del Instituto Nacional de Biodiversidad (INB), de la Universidad Nacional Autónoma de México (MEXU) y del New York Botanical Garden (NY) por permitirme utilizar sus colecciones. A los revisores anónimos por sus comentarios.

\section{Literatura CitADA}

Lellinger, D.B. 1987. The disposition of Trichopteris (Cyatheaceae). Amer. Fern J. 77: 90-94.

Lellinger, D.B. 1989. The ferns and fern-allies from Costa
Rica, Panama, and the Chocó. Part I. Pteridologia 2A: 338-358.

Moran, R.C. 1995. Cnemidaria y Cyathea. In: Davidse, G., M. Sousa \& S. Knapp. (eds.). Flora Mesoamericana. Vol. 1. Psilotaceae a Salviniaceae. Univ. Nac. Autónoma de México. México, D.F. p. 90-103.

Rojas, A.F. 2001. Nuevas especies, nombres nuevamente utilizados y nuevas distribuciones en los helechos arborescentes (Filicales: Cyatheaceae) para el neotrópico. Rev. Biol. Trop. 49: 453-466.

Smith, A.R. \& M.H. Grayum. 1988. Cyathea stolzei $x$ ursina, a distinctive tree fern hybrid from Costa Rica. Amer. Fern J. 78: 105-108.

Stolze, R.G. 1974. A taxonomic revision of the genus Cnemidaria (Cyatheaceae). Fieldiana, Bot. 37: 1-98.

Tryon, R.M. 1970. The classification of the Cyatheaceae. Contr. Gray Herb. 200: 3-53.

Tryon, R.M. 1976. A revision of the genus Cyathea. Contr. Gray Herb. 206: 19-98. 


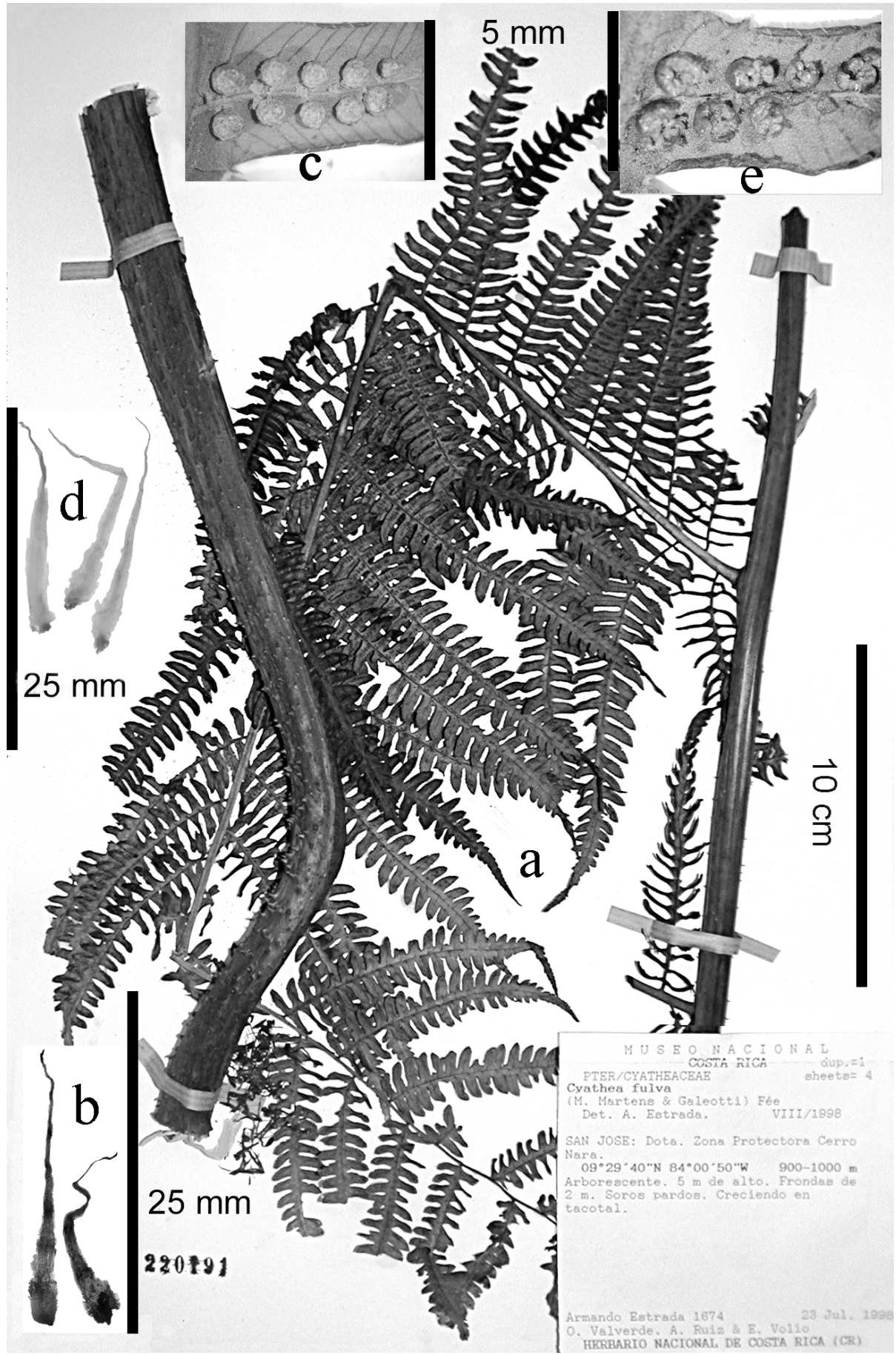

Fig. 1. Ejemplar tipo de Cyathea povedae (A. Estrada et al. 1674, CR): a. Espécimen. b. Escamas del estípite. c. Detalle de la lámina. Cyathea fulva (J. Mickel et al. 5373, MEXU): d. Escamas del estípite. e. Detalle de la lámina. 


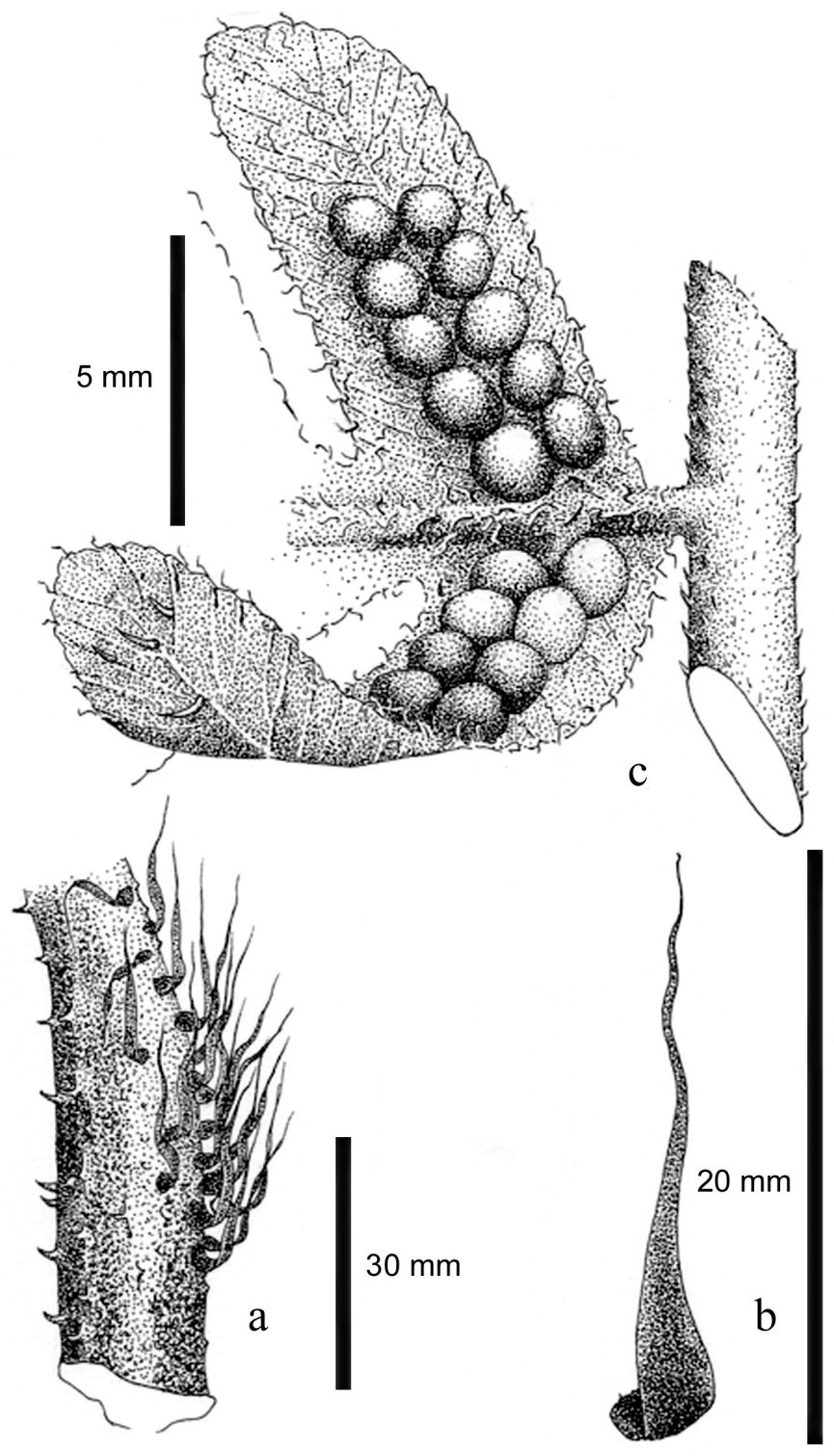

Fig. 2. Cyathea povedae (A. Rojas et al. 4711, INB): a. Base del estípite. b. Escama del estípite. c. Detalle de la lámina. 


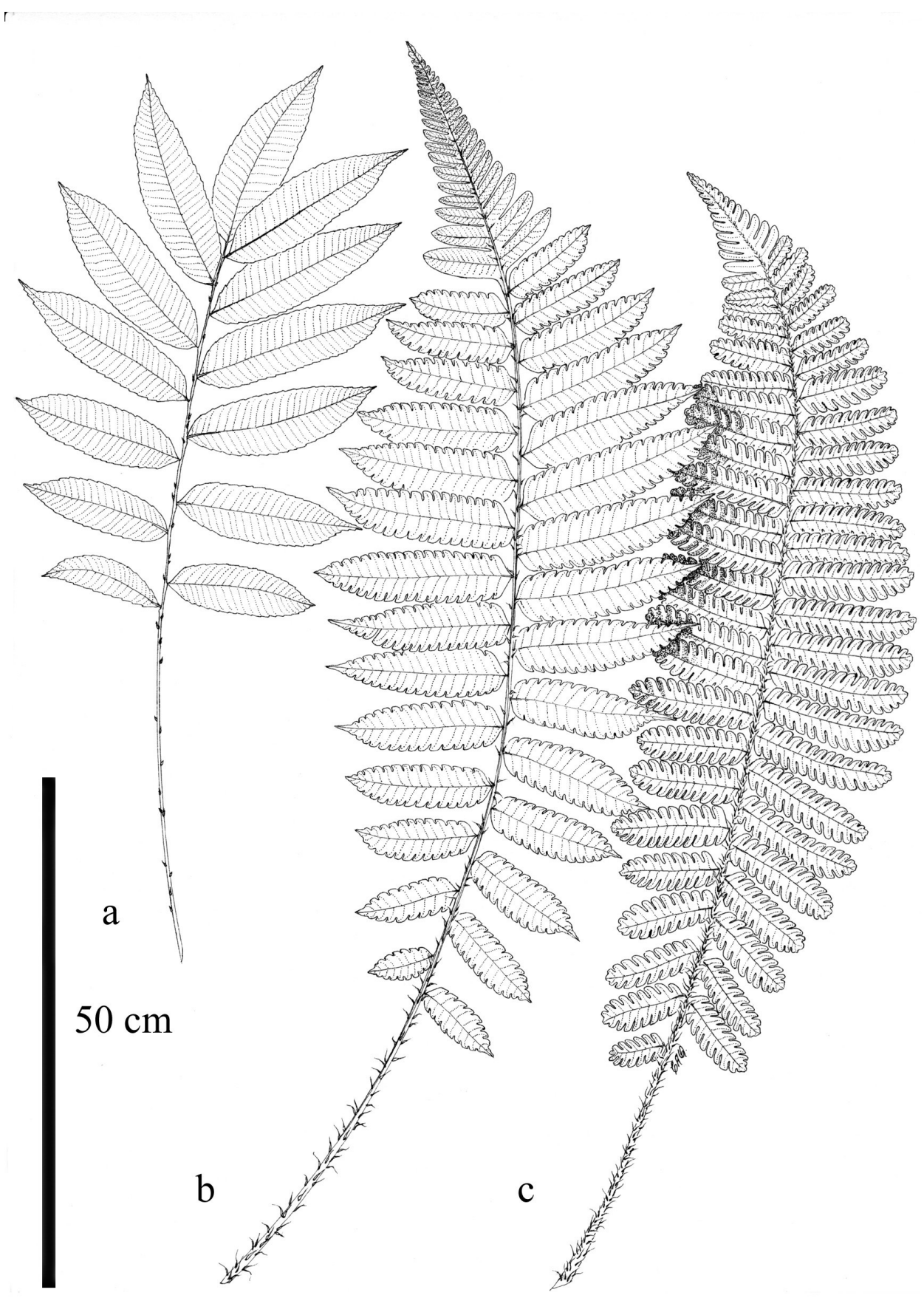

Fig. 3. Fronda de: a. Cyathea stolzei (A. Rojas \& A. Soto 4692, INB). b. C. x smithiana (A. Rojas \& A. Soto 4693, INB). c. C. ursina (A. Rojas \& A. Soto 4691, INB). 

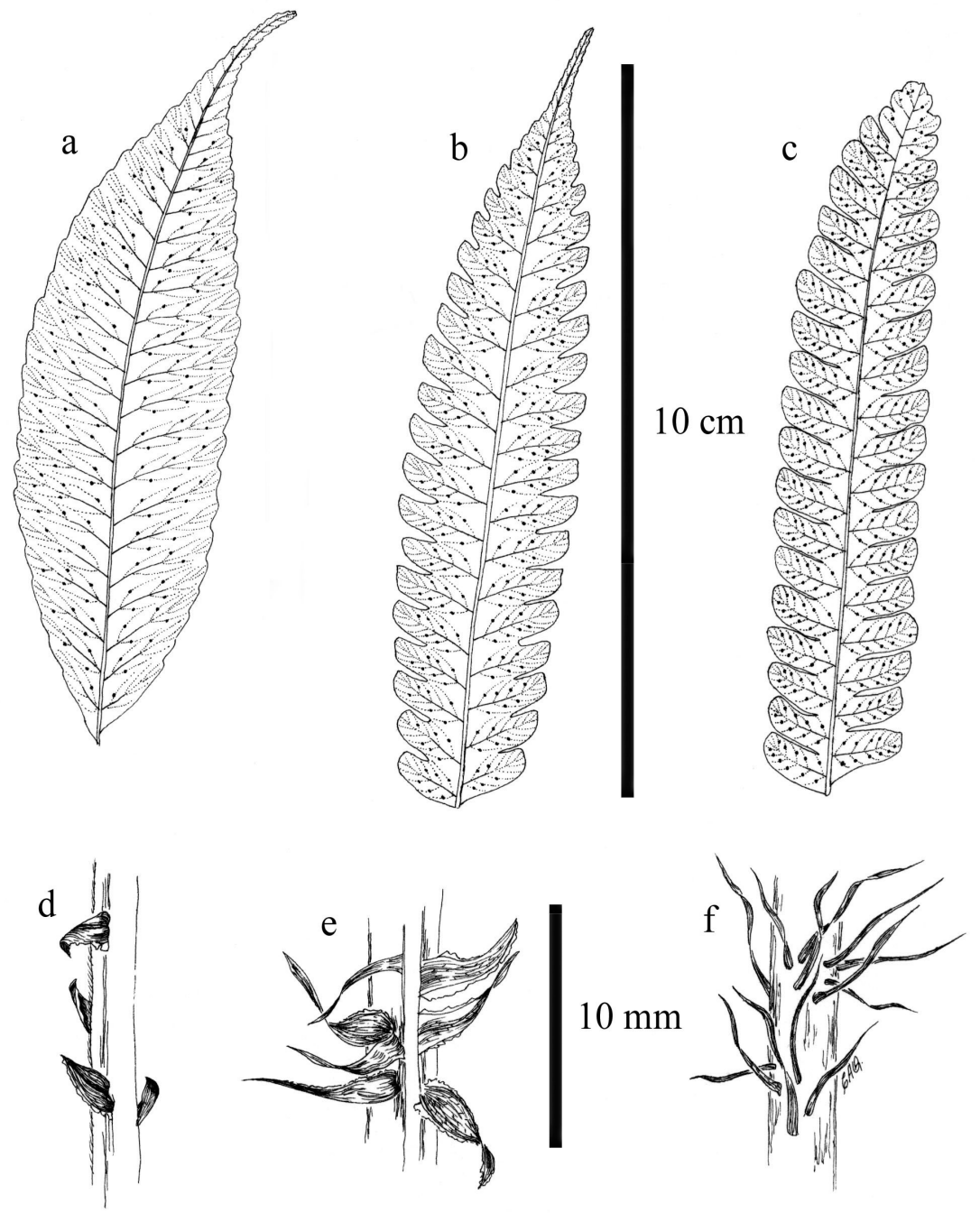

Fig. 4. Pinna media de: a. Cyathea stolzei (A. Rojas \& A. Soto 4692, INB). b. C. x smithiana (A. Rojas \& A. Soto 4693, INB). c. C. ursina (A. Rojas \& A. Soto 4691, INB). Base de los estípites de: d. Cyathea stolzei (A. Rojas \& A. Soto 4692, INB). e. C. x smithiana (A. Rojas \& A. Soto 4693, INB). f. C. ursina (A. Rojas \& A. Soto 4691, INB). 